\title{
A pressure and shear sensor system for stress measurement at lower limb residuum/socket interface
}

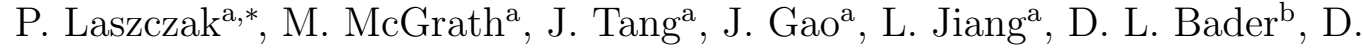 \\ Moser $^{\mathrm{c}}$, S. Zahedi ${ }^{\mathrm{c}}$ \\ ${ }^{a}$ Faculty of Engineering and the Environment, University of Southampton, U.K. \\ ${ }^{b}$ Faculty of Health Sciences, University of Southampton, U.K. \\ ${ }^{c}$ Chas. A. Blatchford 83 Sons Ltd, Endolite Technology Centre, Kingsland Business park, \\ Hampshire RG24 8PZ, U.K.
}

\begin{abstract}
A sensor system for measurement of pressure and shear at the lower limb residuum/socket interface is described. The system comprises of a flexible sensor unit and a data acquisition unit with wireless data transmission capability. Static and dynamic performance of the sensor system was characterised using a mechanical test machine. The static calibration results suggest that the developed sensor system presents high linearity (linearity error $\leq 3.8 \%$ ) and resolution (0.9kpa for pressure and $0.2 \mathrm{kpa}$ for shear). Dynamic characterisation of the sensor system shows hysteresis error of approximately $15 \%$ for pressure and $8 \%$ for shear. Subsequently, a pilot amputee walking test was conducted. Three sensors were placed at the residuum/socket interface of a knee disarticulation amputee and simultaneous measurements were obtained during pilot amputee walking test. The pressure and shear peak values as well as their temporal profiles are presented and discussed.
\end{abstract}

\footnotetext{
*Corresponding Author: Piotr Laszczak, Engineering Materials Research Group, Faculty of Engineering and the Environment, University of Southampton, SO17 1BJ, Southampton, UK; Email, P.Laszczak@soton.ac.uk; Phone, 07513362435
} 
In particular, peak pressure and shear of approximately $58 \mathrm{kPa}$ and $27 \mathrm{kPa}$, respectively, were recorded. Their temporal profiles also provide dynamic coupling information at this critical residuum/socket interface. These preliminary amputee test results suggest strong potential of the developed sensor system for exploitation as an assistive technology to facilitate socket design, socket fit and effective monitoring of lower limb residuum health.

Abstract word count: 200

Keywords: pressure and shear sensor, residuum/socket interface, lower-limb amputees

Manuscript word count: 3395

\section{Introduction}

2

The socket is an essential component of any lower limb prosthesis pro3 viding coupling between the artificial limb and the residuum of the amputee.

4 During daily physical activities, the residuum has to endure intensive and 5 prolonged loading at the residuum/socket interface. Such loads could lead 6 to discomfort, pain or even tissue breakdown. This, in turn, can result in 7 the formation of pressure ulcers (PUs) at the residuum [1], known as stump ulcers. The adverse effects of this condition could lead to periods of bedrest, 9 further surgical intervention, and/or rejection of the prostheses [2]. All of these may be further exacerbated if the socket is poorly fitted.

11 Stresses in both normal (i.e. pressure, $\sigma_{P}$ ) and tangential (i.e. shear, $12 \sigma_{S}$ ) directions exist at the residuum/socket interface, both of which may 13 be detrimental to the soft tissues of the residuum. Indeed, studies suggest that shear may be at least equivalent to pressure as a causative factor for 
soft tissue breakdown [3]. Therefore, its contribution to residuum tissue health should not be understated, particularly considering that shear stresses are unavoidable in both weight-bearing and suspension of the prosthesis [4]. Hence, to minimise the risk of stump ulcers, it is critical to identify those residuum areas which are highly exposed to both pressure and shear.

Nevertheless, most of the current stress monitoring technologies applied at the residuum/socket interface, including a few commercial systems (e.g. Tekscan $^{\mathrm{TM}}[5]$, Novel ${ }^{\mathrm{TM}}[6]$ ), are not sensitive to $\sigma_{S}$. A few tri-axial sensors exist, however they require costly manufacturing techniques [7], or are built on rigid substrates $[8,9,10]$. Rigid sensors have limited compliance with bespoke residuum shapes and hence are not appropriate for use at the residuum/socket interface. Indeed, currently there is lack of a clinicallyfriendly sensor system that can be applied at the residuum/socket interface to simultaneously measure dynamic $\sigma_{P}$ and $\sigma_{S}$.

To address these issues, the authors have recently developed a capacitive interface stress sensor [11], which measures both $\sigma_{P}$ and $\sigma_{S}$ with high linearities and sensitivities. Research lab-based results suggest that this sensor exhibits potential practical advantages, including appropriate spatial resolution, flexibility and thickness. However, no amputee test results were presented. This paper expands on the previous publication by developing a complete sensor system, which is battery operated and features Bluetooth ${ }^{\mathrm{TM}}$ communication, permitting wireless capability. This represents a highly desirable solution in clinical settings, providing the amputees with unconstrained freedom of motion. Furthermore, a pilot amputee test was conducted using the sensor system to obtain real-time biomechanical data. 
Introduction word count: 410

\section{Methods}

\subsection{Sensor system development}

Key components of the interface sensor system include the sensor unit and the data acquisition (DAQ) unit, as illustrated in Fig. 1. The system was designed to measure real-time pressure and shear up to $350 \mathrm{kPa}$ and $60 \mathrm{kPa}$, respectively, during ambulation. Since the sensor units are to be applied at the critical and tight residuum/socket interface, it is also important for the sensors to be flexible and thin to ensure that the comfort of the amputee is not compromised. As detailed in the previous publication [11], a capacitance design principle is employed for the sensor unit which effectively transduces mechanical deformation in the normal and tangential directions to their respective capacitive signals. Adopting the same general principle [11], some improvements were introduced to current sensor unit by utilising silicone as the central mechanical frame material, which exhibits a shore hardness of A20 and a tensile strength of approximately 3.5MPa [12]. This choice of compliant silicone rubber not only ensures comfort of the amputee, but also enables the sensor to conform to residuum local radii of curvatures, typically in the range of $29-83 \mathrm{~mm}$ [13]. Furthermore, differential capacitors $C_{x}$ and $C_{y}$ were adopted to measure x-directional and y-directional shear forces, respectively (Fig. 1). It is also worth noting that, in the current design (Fig. 1 ), the electrodes forming the pressure capacitor $\left(C_{z}\right)$ are separated from the shear electrodes $\left(C_{x}\right.$ and $\left.C_{y}\right)$ to ensure their independent measurements.

A complete DAQ unit was developed, which is capable of $100 \mathrm{~Hz}$ sampling 
and includes 24-bit Digital Signal Processors (DSP) with built-in Capacitanceto-Digital-Converter peripherals, a 32-bit Sensor System Controller (SSC) and a Bluetooth ${ }^{\mathrm{TM}}$ Radio. The sensor unit with the DSPs are connected with the SSC using Flexible Flat Cable (FFC), which is designed to be placed inside the socket. The FFC allows for an exchange of the digitised capacitive measurements between the DSPs and SSC using Serial Peripheral Interface. The SSC is housed within a portable enclosure (approximately $120 \times 80 \times 30 \mathrm{~mm}$ ) which is designed to be worn by the amputee during walking. The signals received by the SSC were subsequently sent to a personal computer (PC) wirelessly via Bluetooth ${ }^{\mathrm{TM}}$. A software, coded in Visual Basic.NET, was developed and installed on the PC, to collect, store and translate the capacitive signals to pressure and shear values based on sensor system calibration.

\subsection{Sensor system calibration}

The sensor system was calibrated using a mechanical test machine (ElectroPuls E1000, Instron Ltd., High Wycombe, UK), which can be used to apply stresses in normal $\left(\sigma_{P}\right)$ and tangential $\left(\sigma_{S}\right)$ directions of the sensor unit, respectively. Both static and dynamic calibration tests were conducted by applying loads equivalent to pressure and shear values up to $350 \mathrm{kPa}$ and $60 \mathrm{kPa}$, respectively. These peak values were chosen as they correspond to maximum values reported at the lower limb residuum/socket interface [14]. The static tests involved the application of incremental loads, each of which was held constant for nine seconds in order to mitigate any transient response. Dynamic loading tests were conducted at frequencies of $1 \mathrm{~Hz}$ and $2 \mathrm{~Hz}$, and involved the application of 100 loading/unloading cycles to the 
sensor unit in a sinusoidal waveform. These frequencies were chosen as they correspond to the frequencies commonly encountered in gait-related events [15]. The dynamic tests' results were then averaged and errors associated with hysteresis were estimated.

\subsection{Pilot amputee test}

A clinical test was conducted by placing multiple sensors at discrete locations of the lower limb residuum and collecting real-time sensor signals. A male amputee with a knee disarticulation (28 years old with a weight of $77 \mathrm{~kg}$ ) participated in the test. The subject, who became an amputee soon after birth and had a re-amputation in his teenage years, used his regular prosthetic components, i.e. supra-condylar suspension socket, KX06 knee and EchelonVT foot (Chas. A. Blatchford \& Sons Ltd., Basingstoke, UK). The subject presented with a healthy, stable residuum, with no signs of soft tissue breakdown and no sensory impairment.

During the tests, the subject wore a silicone gel liner approximately $6 \mathrm{~mm}$ in thickness. After the amputee donned the liner, the sensor units were attached to the outside surface of the liner using double sided tape. Three sensor units were placed at anatomical landmarks of the distal end, anterior proximal and posterior proximal locations of the residuum (Fig. 2a and Fig. 2b), representing primary locations which experience significant stress redistribution during gait [16]. All three sensor units were connected to a single SSC and thus produced synchronised signals. During the tests, each sensor unit produced three channels of signal simultaneously i.e. compression normal to skin $\left(\sigma_{P}\right)$, longitudinal shear $\left(\sigma_{S L}\right)$ and circumferential shear $\left(\sigma_{S C}\right)$, as indicated in Fig. 2c. Prior to the walking tests, all sensor signals in the 
unloaded state were recorded and used as baseline values. Subsequently, the amputee was requested to don the prosthesis and walk at a self-selected pace and cadence (approx. 48 steps/min) on a level surface.

Methods word count: 797

\section{Results}

\subsection{Sensor system calibration}

Fig. $3 \mathrm{a}$ and $3 \mathrm{~b}$ show the static calibration results of the sensor system for pressure and shear, respectively. Strong linear functions are evident between capacitance signal and applied pressure and shear in longitudinal direction, respectively (shear in circumferential direction performs similarly in calibration tests, hence not presented here), and thus were used to convert capacitive readings to appropriate values of $\sigma_{P}$ and $\sigma_{S}$. From the linear fittings in Fig. $3 \mathrm{a}$ and $3 \mathrm{~b}$, linearity errors were estimated as $3.8 \%$ and $1.7 \%$ for pressure and shear channels, respectively. Typical root mean square (RMS) errors for signal noise were approximately $0.35 \mathrm{fF}$, which corresponded to resolutions of $0.9 \mathrm{kPa}$ for the $\sigma_{P}$ and $0.2 \mathrm{kPa}$ for $\sigma_{S}$. It is worth noting that, due to the decoupled electrode design and system compensation, cross-talk between pressure and shear channel was minimized, namely, shear stress output was not affected by pressure. The sensor system also presented negligible effects from bending or stretching when situated at the residuum/socket interface.

The capacitive signals from the dynamic and walking tests were converted to mechanical stresses based on the static calibration results. Fig. 4a and 4b show the converted stress as a function of loading/unloading cycle for pressure and shear stress, respectively. Dynamic hysteresis loops were observed 
in all channels. Hysteresis errors were defined as the maximum difference between the measured stress during loading and unloading $(\Delta \sigma)$ and expressed as a percentage of the former $\left(\sigma_{\text {loading }}\right)$ as indicated in Fig. 4a. As a result, hysteresis errors of approximately $15 \%$ and $8 \%$ were obtained for $\sigma_{P}$ and $\sigma_{S}$, respectively.

\subsection{Pilot amputee test}

Fig. 5 presents measured $\sigma_{P}, \sigma_{S L}$ and $\sigma_{S C}$ over a few exemplary gait cycles from the sensors at the distal end, anterior proximal and posterior proximal locations. All measured stresses during the amputee test show cyclic patterns. Peak $\sigma_{P}$ values of $58 \mathrm{kPa}, 38 \mathrm{kPa}$ and $36 \mathrm{kPa}$ were estimated at the distal end, posterior proximal and anterior proximal locations, respectively. Peak $\sigma_{S L}$ value at the distal end was observed to be $27 \mathrm{kPa}$ and peak $\sigma_{S C}$ was approximately $1.5 \mathrm{kPa}$ (Fig. 5a). $\sigma_{S C}$ values at the anterior proximal and posterior proximal locations were below $1 \mathrm{kPa}$ and therefore are not shown in Fig. $5 \mathrm{~b}$ and $5 \mathrm{c}$.

Results word count: 354

\section{Discussion}

\subsection{Sensor system calibration}

The static calibration results shown in Fig. 3 suggest that the sensor system exhibits high linearity (approx. $3.8 \%$ for $\sigma_{P}$ and $1.7 \%$ for $\sigma_{S}$ ), corresponding to high resolutions of approximately $0.9 \mathrm{kPa}$ for $\sigma_{P}$ and $0.2 \mathrm{kPa}$ for $\sigma_{S}$, respectively. High linearity is advantageous for post-signal processing as it can potentially lead to simplified signal conditioning and system calibration. The linearity and resolution from our sensor system represent an 
improvement on values previously published for commercial, pressure only measuring systems, such as Tekscan ${ }^{\mathrm{TM}}$ with $6 \%$ linearity [17] and $2 \mathrm{kPa}$ resolution (sensor 9811E [18] and ELF electronics [19]). In addition, our sensor system provides synchronised shear transduction, which is essential for mechanical stress sensing at the residuum/socket interface [3]. In order to measure both pressure and shear stresses at the lower limb residuum/socket interface, a strain-gauge based sensor system has been reported as one of the few systems applied at this critical interface. Nonetheless, a linearity error (2.11\% [10]) and resolutions $\left(0.33 \mathrm{kPa}\right.$ for $\sigma_{P}$ and $0.15 \mathrm{kpa}$ for $\left.\sigma_{S}[10]\right)$ were reported for the strain gauge based system which are similar to those obtained from our system. However, it is worth noting that the reported strain gauge sensors were of large dimensions (47mm [14]) and hence can only be mounted outside the socket. This not only required a degree of socket rectification, but also induced potential interference with the walking tests. Furthermore, it was reported that a precise insertion of the strain gauge sensors at the residuum/socket interface was required, as the transducer protrusion above the socket wall may also affect the measurements of stresses per se. Indeed, literature on similar sensors suggests that even $1.6 \mathrm{~mm}$ protrusion may cause errors of up to $85 \%$ [20]. All these constraints can limit the potential application of this technology in clinical and home settings. In contrast, in our sensor system, the sensor unit along with the FFCs are thin and flexible, and thus can be directly placed inside the amputee's socket without any socket modification. This also enables dynamic pressure and shear measurement directly from the residuum/socket interface, reflecting real-time load transfer at this critical interface. Furthermore, the usage of portable SSC unit along 
with wireless communication for data transmission allows unconstrained ambulation during amputee test. It is also worth noting that a pressure value of $<8 \mathrm{kPa}$ could cause soft tissue ischemia [21] and shear may be at least equivalent to pressure as an external factor affecting tissue breakdown [3]. Therefore, the high resolution of pressure and shear measurements estimated from the present sensor system offers promise for potentially providing an early indication of tissue breakdown at the residuum.

The dynamic results in Fig. 4 reveal typical hysteresis loops between the loading and unloading cycles. Hysteresis errors of $15 \%$ and $8 \%$ were obtained for $\sigma_{P}$ and $\sigma_{S}$, respectively. This may be attributable to the viscoelastic mechanical behaviour of the middle silicone layer used in the sensor unit. Hysteresis is commonly observed for thin and flexible interface sensor designs. For example, the Tekscan ${ }^{\mathrm{TM}}$ system was reported to exhibit $24 \%$ hysteresis error for pressure alone [22], which is higher than that associated with the present system. Nonetheless, we envisage that the hysteresis in our sensor system could be potentially compensated and improved by incorporating appropriate mechanical modelling in the DAQ unit, which will be included in future work.

\subsection{Pilot amputee test}

Fig. 5a shows repetitive $\sigma_{P}$ patterns from the sensor positioned at the distal end and the typical peak $\sigma_{P}$ value is approximately $58 \mathrm{kPa}$ (peak values for different steps ranged $\pm 7 \mathrm{kPa}$ ). Only a few studies have reported $\sigma_{P}$ measurements from similar anatomical regions. For example, Lee [16] reported $38 \mathrm{kPa}$ mean change of $\sigma_{P}$ in the distal region for a subject of $80 \mathrm{~kg}$ in weight. Kahle [23] reported $\sigma_{P}$ for the distal region in the range of $15-61 \mathrm{kPa}$ for a 
group of lower limb amputees. The variability of the $\sigma_{P}$ can be attributed to the wide range of subject weights (i.e. $55-115 \mathrm{~kg}$ ), different socket fits, residuum geometries and prosthetic components etc. [23]. Our measured $\sigma_{P}$ values are of similar magnitude to these reported data. The pressure temporal profile (Fig. 5a) partially resembles the double hump form as characteristic of a vertical component of ground reaction force (GRF) in amputee gait [24]. Within each cycle, we also observe that the interface $\sigma_{P}$ shows a more gradual increase, followed by a slower decrease as in comparison with profile of a typical vertical GRF [24]. This may reflect the viscoelastic nature of soft tissues at the residuum/socket interface, which may promote much more damped features of the stress temporal profiles. Such interface stress details in both early stance/loading and in swing/unloading phases would not have been possible to be shown by detecting vertical GRF alone. In addition, a peak value of $27 \mathrm{kPa}$ (ranging $\pm 4 \mathrm{kPa}$ between steps) was obtained for $\sigma_{S L}$ from the distal end. To the best of our knowledge, only one other study investigated shear stresses at the residuum of trans-femoral amputees [25], and reported shear stresses of up to $24 \mathrm{kPa}$. Furthermore, within each cycle, at early stance phase, $\sigma_{S L}$ increases more rapidly with higher stress rates (approximately $170 \mathrm{kPa} / \mathrm{s}$ ) as compared to that of $\sigma_{P}$ (approximately $110 \mathrm{kPa} / \mathrm{s}$ ), which shows more gradual changes (Fig. 5a). Given the reported relationship between the applied shear rate and development of lesions at the residuum [26], such real-time detection of shear rate at the residuum/socket interface could be useful to monitor health of the residuum. We envisage that the detected changes in interface loading profiles over time could be potentially utilised to clinically assess, for example, socket fit, gait and overall 
rehabilitation outcome. In contrast to $\sigma_{P}$ and $\sigma_{S L}$, low value of $\sigma_{S C}$ in the range up to $1.5 \mathrm{kPa}$ was obtained. Nonetheless, it is worth noting that, $\sigma_{S C}$ acts in the medial direction (i.e. negative), but decreases during the stance phase, indicating small medial-lateral movement during stance. Furthermore, $\sigma_{S C}$ may also increase during turning manoeuvres.

For the posterior proximal location, a peak value of approximately $38 \mathrm{kPa}$ ( $\pm 5 \mathrm{kPa})$ was obtained for $\sigma_{P}$, as shown in Fig. 5b. Pressures in the range of $36-59 \mathrm{kPa}$ at similar locations have been previously reported [16] for transfemoral amputees of similar weight $(80 \mathrm{~kg}) . \sigma_{P}$ also shows a double hump temporal profile, with the second peak greater than the first one. This may be associated with the residuum engaging with posterior socket wall, as the amputee propels himself throughout the mid- to late-stance phase. Peak values of $\sigma_{S L}$ reach up to approximately $5 \mathrm{kPa}$ at this posterior proximal site. The only other study, which investigated shear stresses at the trans-femoral residuum, reported longitudinal shear of $3 \mathrm{kPa}$ or less at the majority of the posterior locations [25]. The relatively small values of $\sigma_{S L}$ are believed to originate from a host of factors, including large contact area of the socket, high volume of residuum soft tissues or presence of lubricant (e.g. sweat) [25], all of which promote even distribution of stresses. It is also evident that, within each cycle, $\sigma_{S L}$ shows shear towards the proximal direction (i.e. positive) in stance phase and shear towards the distal direction (i.e. negative) in swing, which briefly follows the 'pistoning' effect i.e. a strong dynamic coupling between the residuum and the socket along the longitudinal axis of the prosthesis [27]. It is noteworthy that $\sigma_{S L}$ at the posterior proximal location decreases after an initial peak (Fig. 5b). This aligns with the characteristic 
reduction in gluteus maximus activity [28], the relaxation of which may reduce compliance of the muscle resulting in reduction of $\sigma_{S L}$ at the posterior location. For the anterior proximal location, Fig. 5c shows that $\sigma_{P}$ and $\sigma_{S L}$ generally follow similar patterns as those from the posterior proximal location. However, $\sigma_{S L}$ at the former location does not change direction to distal in swing phase, but remains low (up to $1 \mathrm{kPa}$ ) instead.

Synchronised $\sigma_{P}$ measurements from all three anatomic locations are compared in Fig. 5d. Typical $\sigma_{P}$ peak values $(58 \mathrm{kPa})$ at the distal end are considerably higher than those from proximal locations $(38 \mathrm{kPa}-36 \mathrm{kPa})$ as the distal end is subjected to more localised weight bearing. This effect can be exacerbated by the brimless socket worn by the amputee during the tests. Fig. 5d also demonstrates that at the posterior proximal location, which typically provides a 'seating' interface for the amputees, $\sigma_{P}$ exhibited second peak higher than the first one, while $\sigma_{P}$ at the anterior proximal significantly reduced after the first peak. The latter effect may reflect that the subject propels against the posterior wall, thereby off-loading the anterior location.

The results of the pilot walking test provide preliminary validation of our sensor system for measuring dynamic pressure and shear stresses at the lower limb residuum and the prosthetic socket interface. This is despite that the geometries of trans-femoral sockets are known to cause many technical difficulties for interface measurements, which may prohibit sensor measurements. Indeed, stress measurements at the distal region are frequently constrained for transducers mounted within socket wall $[10,14]$, due to interference with prosthetic knee mechanism, while fluid-filled sensors have been reported to exhibit errors when their tubing was too long [29]. To the best of our knowl- 
edge, simultaneous measurements of $\sigma_{P}$ and $\sigma_{S}$ on trans-femoral amputees have not been reported previously. Future works will include more interfacial pressure and shear data collection from more amputee subjects which, we believe, could shed light on biomechanical coupling at the residuum/socket interface for both trans-femoral and trans-tibial amputees. It is also worth noting the possible limitations of this study, including current lack of outcome measures involving, for example, socket comfort and amputee gait, repeatability and accessibility of the sensor data, as well as durability and usability of the device in real clinical settings, each of which will be examined in future work.

Discussion word count: 1600

\section{Conclusions}

A novel interface pressure and shear sensor system was developed with a view to monitoring the dynamic stresses at the lower limb residuum/socket interface. The performance and clinical effectiveness of the system were initially examined in a pilot amputee test, i.e. by mounting three sensors at key anatomical landmarks of the residuum. Real-time outputs from our sensor system not only produced peak stresses which align with values reported in the literature, but also provided detailed temporal profiles of interfacial pressure and shear stress at the residuum. Such interfacial load transfer information would not have been possible to be obtained by using conventional means of ground reaction force measurements alone. The sensor system was also designed to ensure amputee's comfort and overall user friendliness by adopting a wireless approach for data communication. The developed sensor 
system is the first of its kind that simultaneously measures both pressure and shear and is preliminarily proven to be applicable directly at lower limb residuum/socket interface without any alteration to the existing socket. Such a system could be utilized to provide objective measures of socket design, comfort and fit as well as long-term monitoring of outcomes of prosthesis usage and rehabilitation, potentially transforming current clinical practice. The in-situ pressure and shear measurement can also provide essential load inputs for models designed to predict tissue tolerance levels at the loaded residuum/socket interface, with the potential of increased biomechanical understanding and knowledge.

Conclusions word count: 235

\section{Acknowledgements}

Competing interests: None declared

Project co-funded by the University of Southampton and Chas A Blatchford \& Sons Ltd.

Ethical approval: This study was approved by University of Southampton Ethics and Research Governance Committee (ID: 6008) 
[1] Dudek NL, Marks MB, Marshall SC, Chardon JP. Dermatologic conditions associated with use of a lower-extremity prosthesis. Arch Phys Med Rehabil 2005;86(4):659-63.

[2] Bader DL, Bouten C, Colin D, Oomens CWJ. Pressure ulcer research; chap. 4. 1st ed.; Berlin: Springer-Verlag; 2005, p. 35-6.

[3] Zhang M, Roberts VC. Effect of shear forces externally applied to skin surface on underlying tissues. J Biomed Eng 1993;15(6):451-6.

[4] Zhang M, Turner-Smith AR, Roberts VC, Tanner A. Frictional action at lower limb/prosthetic socket interface. Med Eng Phys 1996;18(3):20714.

[5] Dumbleton T, Buis AW, McFayden A, McHugh BF, McKay G, Murray KD, et al. Dynamic interface pressure distributions of two transtibial prosthetic socket concepts. J Rehabil Res Dev 2009;46(3):405-15.

[6] Boutwell E, Stine R, Hansen A, Tucker K, Gard S. Effect of prosthetic gel liner thickness on gait biomechanics and pressure distribution within the transtibial socket. J Rehabil Res Dev 2012;49(2):227-40.

[7] Sundara-Rajan K, Bestick A, Rowe GI, Klute GK, Ledoux WR, Wang $\mathrm{HC}$, et al. An interfacial stress sensor for biomechanical applications. Meas Sci Technol 2012;23(8):1-10.

[8] Razian M, Pepper MG. Design, development, and characteristics of an in-shoe triaxial pressure measurement transducer utilizing a single element of piezoelectric copolymer film. IEEE Trans Neural Syst Rehabil Eng 2003;11(3):288-93. 
[9] Wiliams RB, Porter D, Roberts VC, Regan JF. Triaxial force transducer for investigating stresses at the stump/socket interface. Med Biol Eng Comput 1992;30(1):89-96.

[10] Sanders JE, Daly CH. Measurement of stresses in three orthogonal directions at the residual limb-prosthetic socket interface. IEEE Trans Rehabil Eng 1993;1(2):79-85.

[11] Laszczak P, Jiang L, Bader DL, Moser D, Zahedi S. Development and validation of a 3D-printed interfacial stress sensor for prosthetic applications. Med Eng Phys 2015;37(1):132-7.

[12] Bluestar Silicones. Silbione RTV 4020 datasheet. [Online]; 2012. http: //www. silbione. com/wp-content/uploads/2014/01/RTV-4420. pdf.

[13] Schreiner RE, Sanders JE. A silhouetting shape sensor for the residual limb of a below-knee amputee. IEEE Trans Rehabil Eng 1995;3(3):24253.

[14] Sanders JE, Zachariah SG, Jacobsen AK, Fergason JR. Changes in interface pressures and shear stresses over time on trans-tibial amputee subjects ambulating with prosthetic limbs: comparison of diurnal and six-month differences. J Biomech 2005;38(8):1566-73.

[15] Antonsson EK, Mann RW. The frequency content of gait. J Biomech 1985;18(1):39-47.

[16] Lee VSP, Solomonidis SE, Spence WD. Stump-socket interface pressure as an aid to socket design in prostheses for trans-femoral amputees - a preliminary study. Proc Inst Mech Eng H 1997;211(2):167-80. 
[17] Tekscan . Flexiforce sensors manual. [Online]; 2010. http://www . tekscan.com/pdf/FLX-FlexiForce-Sensors-Manual.pdf.

[18] Tekscan . Medical sensor 9811e. [Online]; 2015. https: //www.tekscan.com/sites/default/files/resources/ MDL-Medical-Sensor-9811E-Datasheet.pdf.

[19] Tekscan . Elf system manual. [Online]; 2015. https: //www.tekscan.com/sites/default/files/resources/ELF\%20User\% 20Manual\%20RevN.pdf.

[20] Appoldt FA, Bennett LM, R. C. Socket pressure as a function of pressure transducer protrusion. Bull Prost Res 1969;10-11:236-49.

[21] Halloway GA, Daly CH, Kennedy D, Chimoskey J. Effects of external pressure loading on human skin blood flow measured by ${ }^{133} \mathrm{Xe}$ clearance. J Appl Physiol 1976;40(4):597-600.

[22] Polliack AA, Sieh RC, Craig D, Landsberger S. amd McNeil R, Ayyappa E. Scientific validation of two commercial pressure sensor systems for prosthetic socket fit. Prosthet Orthot Int 2000;24(1):63-73.

[23] Kahle JT, Highsmith MJ. Transfemoral sockets with vacuum-assisted suspension comparison of hip kinematics, socket position, contact pressure, and preference: Ischial containment versus brimless. J Rehabil Res Dev 2013;50(9):1241-52.

[24] Perry J, Burnfield J. Gait Analysis: Normal and Pathological Function; chap. 1,22. 2nd ed.; Thorofare, NJ: SLACK Incorporated; 2010,. 
[25] Appoldt FA, Bennett LM, R. C. Tangential pressure measurements in above knee sockets. Bull Prosthet Res 1970;10(13):70-86.

[26] Naylor PFD. Experimental frictional blisters. Br J Dermatol 1955;67(10):327-42.

[27] Tang J, McGrath M, Laszczak P, Jiang L, Bader D, Moser D, et al. Characterisation of dynamic couplings at lower limb residuum/socket interface using 3D motion capture. Med Eng Phys 2015;37(12):1162-8.

[28] Wentink EC, Prinsen EC, Rietman JS, Veltink PH. Comparison of muscle activity patterns of transfemoral amputees and control subjects during walking. J Neuroeng Rehabil 2013;10(87).

[29] van Pijkeren T, Naeff M, Kwee HH. A new method for the measurement of normal pressure between amputation residual limb and socket. Bull Prosthet Res 1980;10-33:31-4. 

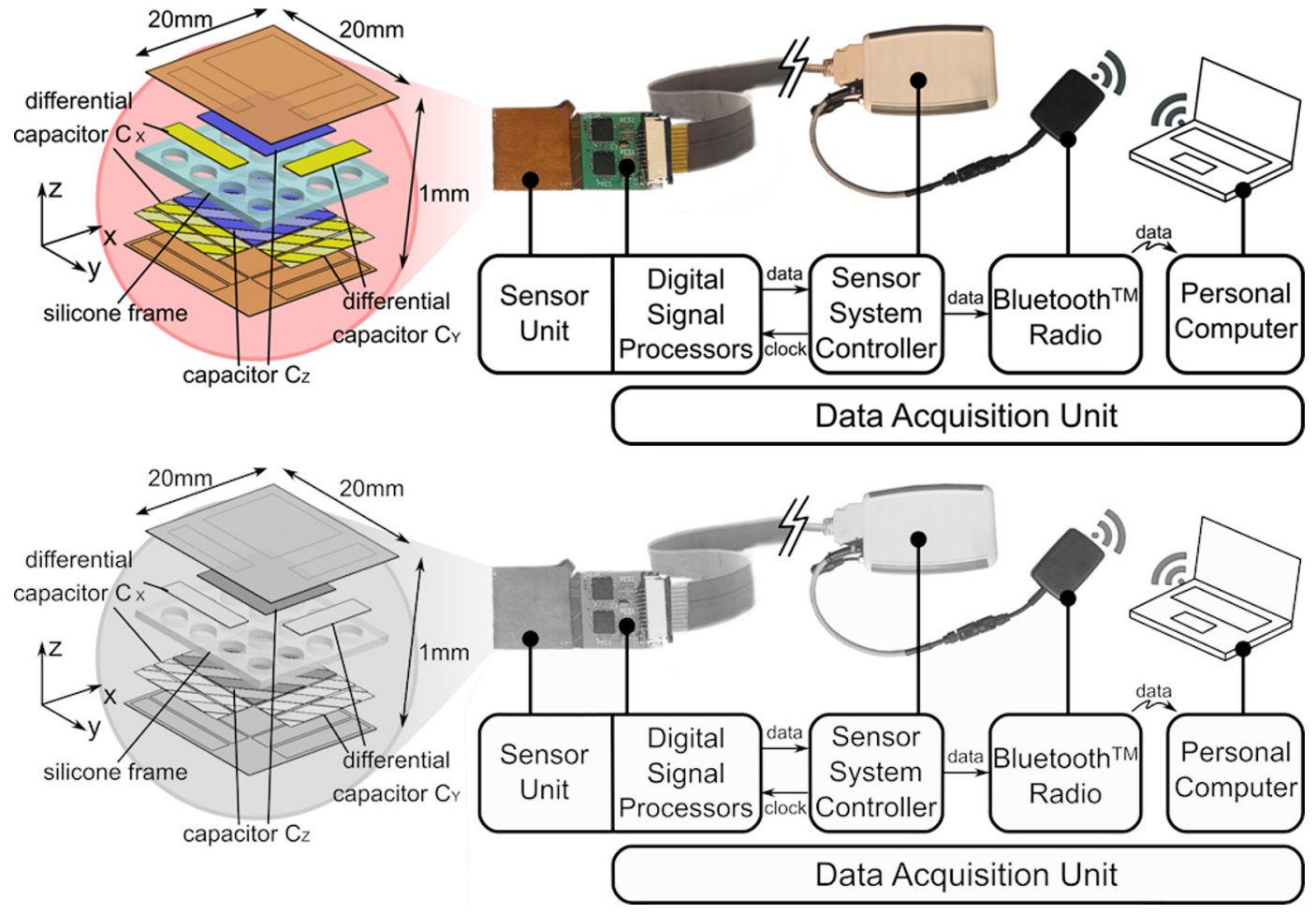

Figure 1: Key components of the sensor system, which include sensor unit and the data acquisition unit, consisting of Digital Signal Processors, Sensor System Controller, Bluetooth $^{\mathrm{TM}}$ Radio and Personal Computer with custom software. 

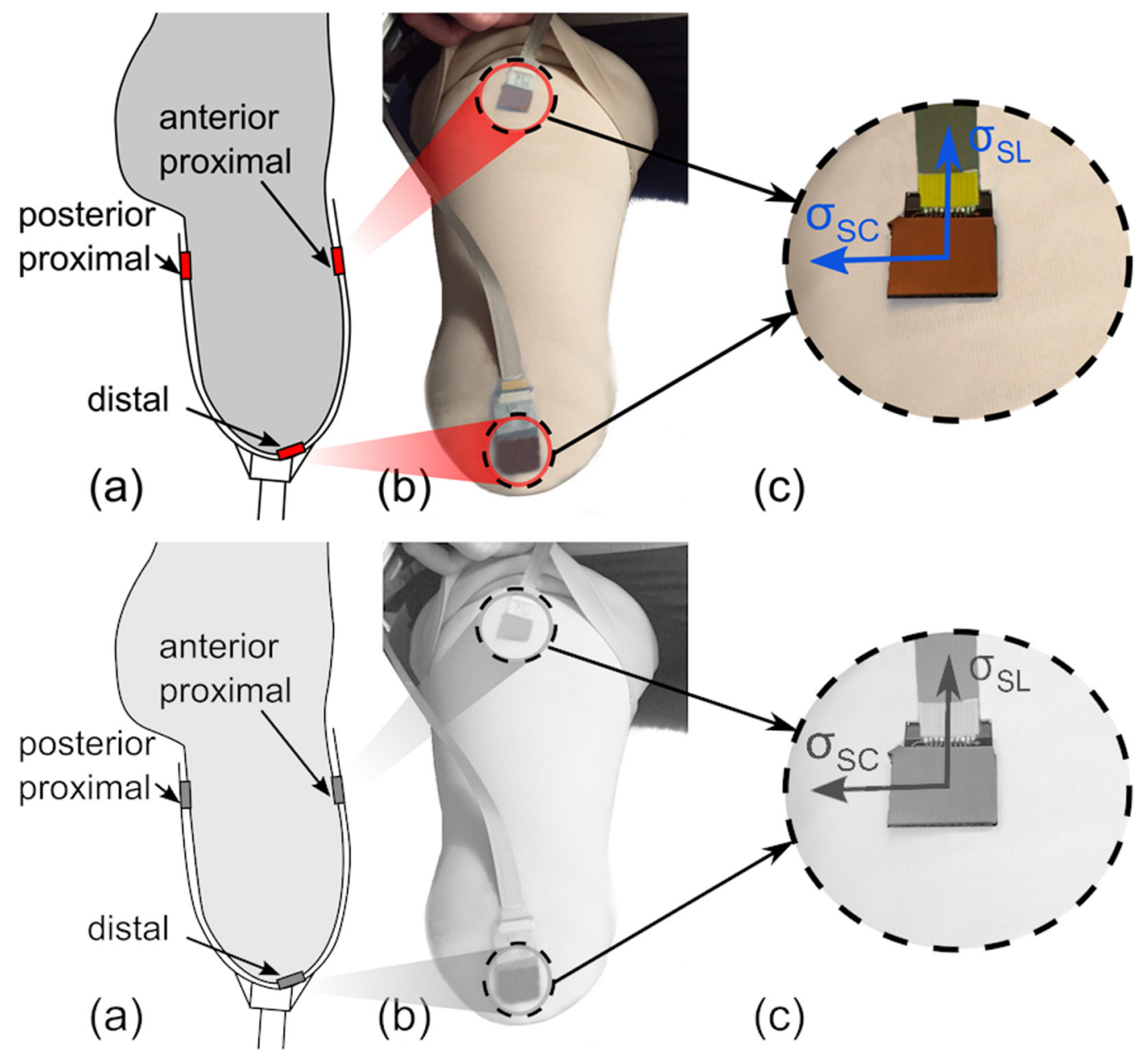

Figure 2: (a) Schematic drawing showing sites where the three sensors were placed. (b) Image of the distal and anterior proximal sensors located at the residuum. (c) Coordinate system showing directions of $\sigma_{S L}$ (longitudinal shear, proximal positive) and $\sigma_{S C}$ (circumferential shear, lateral positive). 

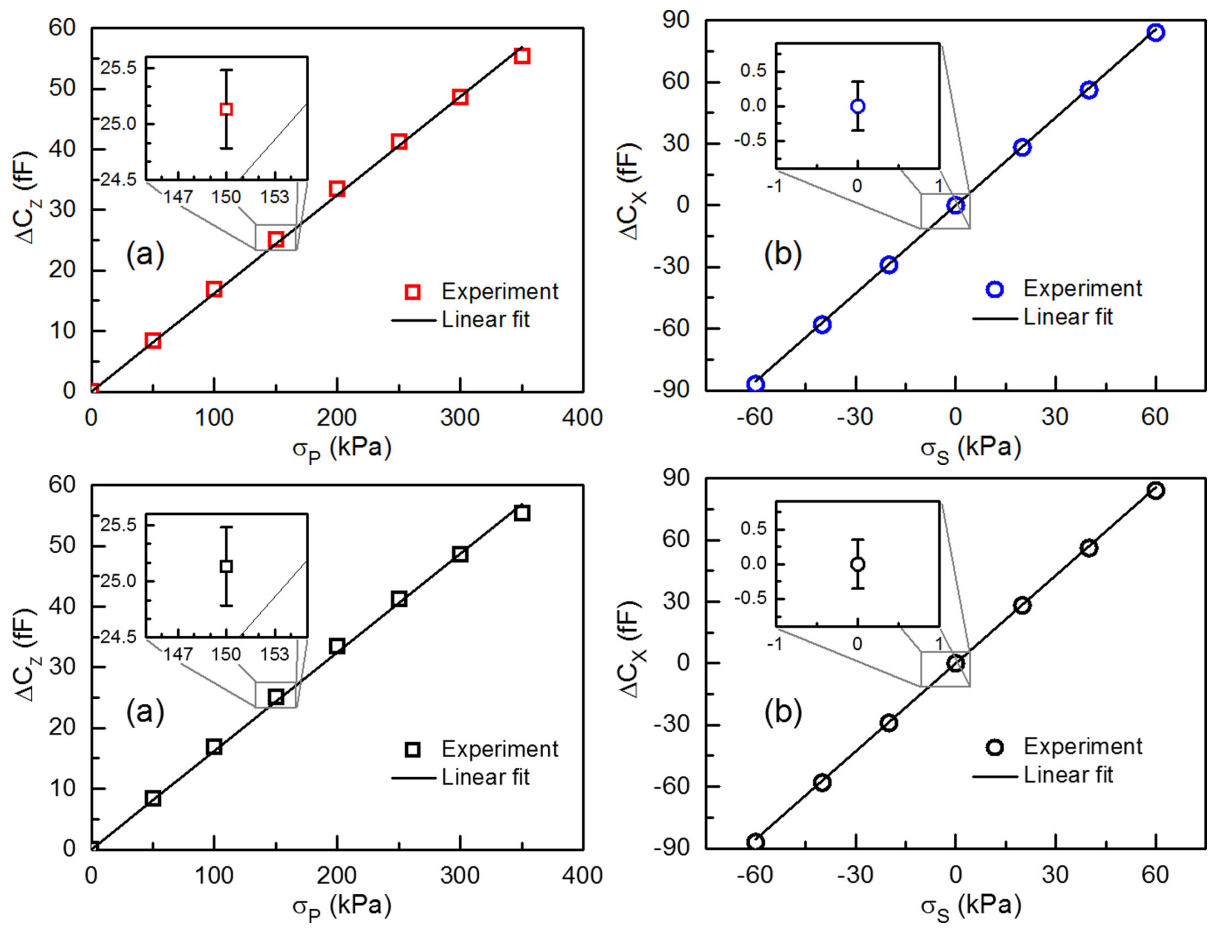

Figure 3: The static calibrations of the sensor for (a) pressure $\left(\sigma_{P}\right)$ and (b) shear $\left(\sigma_{S}\right)$ sensing channels. Symbols represent experimental data and solid lines represent linear fittings. Error bars correspond to root mean square of noise. 

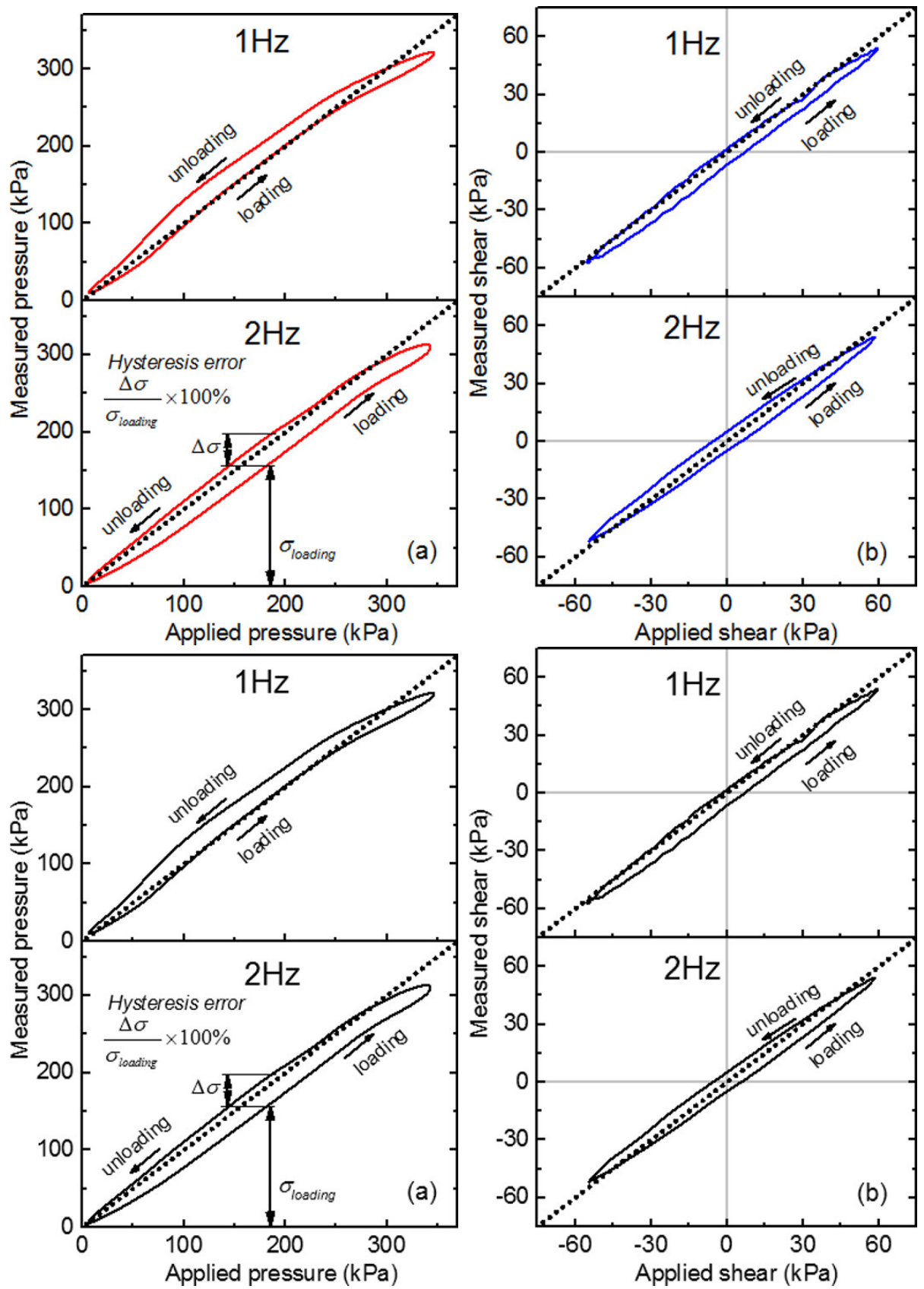

Figure 4: The hysteresis loops for $1.0 \mathrm{~Hz}$ (top) and $2.0 \mathrm{~Hz}$ (bottom) dynamic loading/unloading test for (a) pressure $\left(\sigma_{P}\right)$ and (b) shear $\left(\sigma_{S}\right)$ sensing channels. Dotted lines in each figure signify values where applied stresses are equal to measured stresses. 

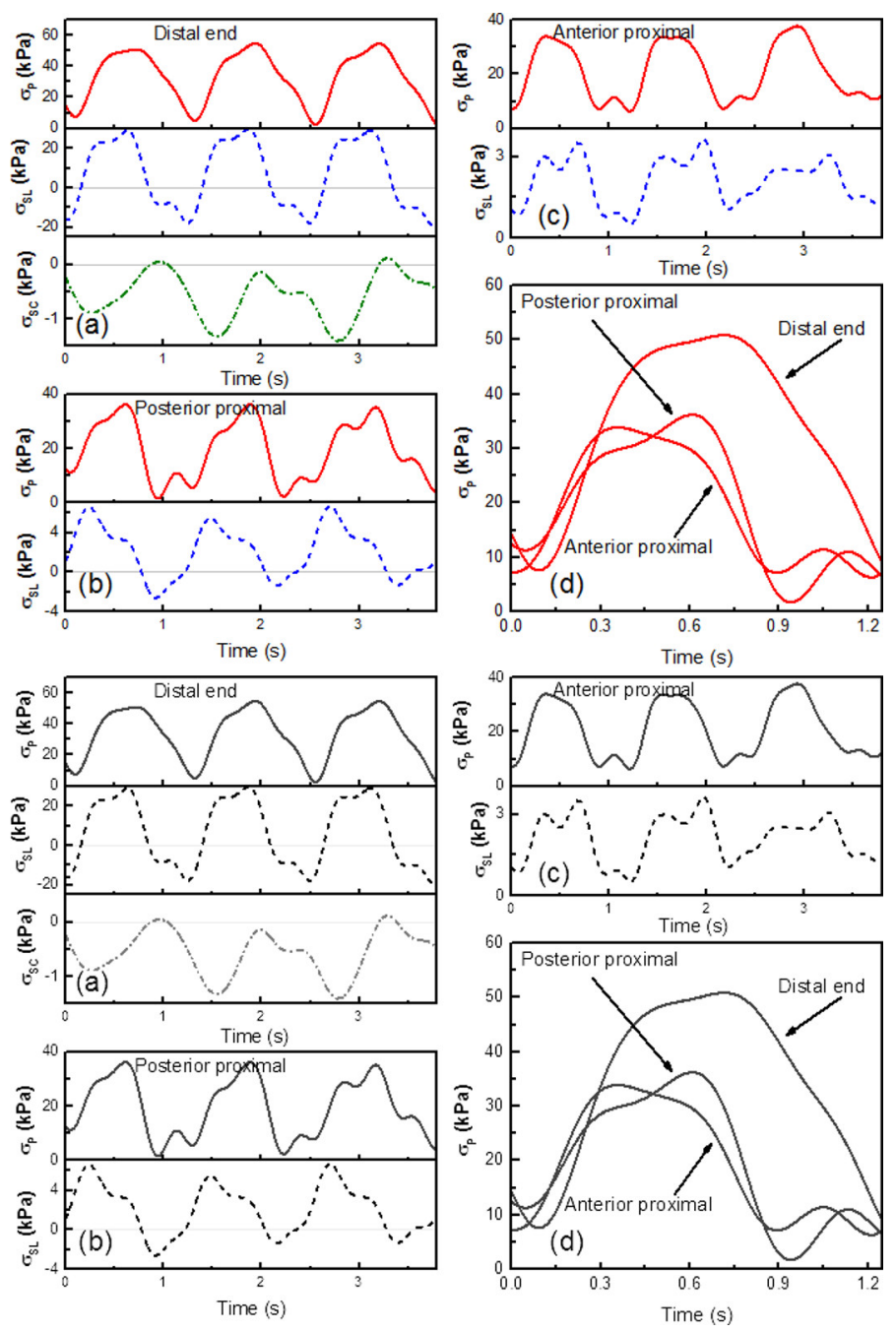

Figure 5: Measured interfacial stresses as a function of time from sensors positioned at (a) distal end (b) posterior proximal and (c) anterior proximal, respectively. A comparison of pressure measurements at all three locations is shown in (d). 